\title{
Jejunojejunal Intussusception After Roux-en-Y Gastric Bypass: A Review
}

\author{
Loic Daellenbach • Michel Suter
}

Published online: 15 October 2010

(C) Springer Science+Business Media, LLC 2010

\begin{abstract}
Small bowel intussusception is a rare long-term complication after Roux-en-Y gastric bypass, the etiology of which remains unclear. Except for one series reporting on 23 patients, case reports represent the vast majority of all cases reported so far. With this complete review of the world literature, based on a total of 63 patients including 2 of our own cases, we provide an extensive overview of the subject. The origin of intussusception after gastric bypass is different from that of intussusception of other causes, in that there is usually no lead point. It is likely related to motility disorders in the divided small bowel, especially in the Roux limb. This rare condition may cause obstruction and lead to bowel necrosis if not recognized and treated promptly. Clinical presentation is not specific. Computerized tomography scan represents the diagnostic test of choice, but surgery is sometimes the only way to establish the diagnosis. Treatment may be limited to reduction if the small bowel is viable, but resection of the affected segment is recommended on the basis of this review, since it seems to result in fewer recurrences. Knowledge of this entity and a high index of suspicion are required to make the correct diagnosis and offer appropriate treatment in a timely fashion.
\end{abstract}

\section{Daellenbach}

Faculty of Medicine and Biology, University of Lausanne,

Lausanne, Switzerland

M. Suter $(\bowtie)$

Department of Surgery, Hôpital du Chablais,

1860 Aigle, Switzerland

e-mail: michelsuter@netplus.ch

\section{Suter}

Department of Visceral Surgery,

Centre Hospitalier Universitaire Vaudois,

Lausanne, Switzerland
Keywords Gastric bypass · Complications · Intussusception

\section{Introduction}

The prevalence of obesity is rapidly increasing worldwide. In the USA, between $25 \%$ and $30 \%$ of the adult population is obese, and a growing percentage of these patients have severe or morbid obesity. In Europe, this figure lies between $8 \%$ and $23 \%$ and $9 \%$ in Switzerland, but the prevalence is also growing rapidly. For patients with morbid obesity, or severe obesity associated with comorbidities, bariatric surgery is the only efficient long-term treatment option. Currently, more than 300,000 procedures are performed worldwide annually [1]. Roux-en-Y gastric bypass (RYGBP) still represents the operation of choice for many bariatric surgeons and the "gold standard" to which other procedures need to be compared. Long-term complications are not uncommon after gastric bypass and lead to reoperation in $3-20 \%$ of the patients. Bowel occlusion or chronic recurrent abdominal pain related to internal hernia or adhesions account for most of them.

Intussusception represents an uncommon cause of symptoms after RYGBP. In children, intussusception is not exceptional. It usually affects the ileo-caecal area and is caused by lymphoid hyperplasia in the distal ileum. In adults, small bowel intussusception is rare, and it is associated in almost all cases with some pathological area in the small bowel, like a polyp, which acts as a so-called lead point. In most cases, it develops along with peristalsis, and the proximal segment invaginates into the distal one (antegrade intussusception). Intussusception can develop after RYGBP in a small subgroup of patients without any lead point and can cause recurrent abdominal pain, obstruction and/or bowel ischemia, and necrosis. The pathophysiology of intussusception in this setting remains 
poorly understood although motility disorders following construction of the Roux limb have been postulated by some to play a major role. Intussusception after gastric bypass is almost always retrograde although a few anterograde cases have been reported.

In this paper, we provide a complete review of the existing medical literature on intussusceptions after gastric bypass and summarize the current knowledge on this unusual complication. Despite its rarity, intussusception needs to be included in the differential diagnosis of long-term abdominal pain and small bowel occlusion after RYGBP so that adequate diagnostic and treatment options can be considered early enough.

\section{Patients and Methods}

This review is based on a complete literature search in the Medline/PubMed database using the keywords "intussusception" and "gastric bypass", completed by manual search using reference lists from individual articles. This review included all English/French/German literature and all other languages provided that an abstract was available. Patients were included if they had undergone an open or laparoscopic Roux-en-Y gastric bypass and later developed an antegrade or retrograde small bowel intussusception as proven by surgical exploration, or highly suggested by radiological imaging.

A total of 61 patients were found in 24 articles [2-27], the majority of which were case reports. Some of these patients were included in papers focusing on intestinal obstruction of various origins, with only few details provided on presentation and treatment. One single group, however, reported on a series of 23 patients in 2008 [2], and more recently presented an extension of their experience, with the addition of 16 new cases, in the form of a poster [3]. As this group's experience represents more than half the published cases, one of the authors (SC Simper) was contacted for additional information about their experience. Two patients encountered in our own experience are also described and one of them is also included in the analysis.

Our analysis focused on the general and morphological characteristics of the patients and on the delay and weight loss between original surgery and Intussusception. We also evaluated the clinical presentation of the latter, with physical, radiological, and surgical findings, as well as treatment modalities and outcome.

\section{Results}

\section{Literature Review}

Table 1 summarizes findings from all patients described in the literature, with the addition of our own two cases. Some reports unfortunately do not provide the reader with all the necessary details about the patient(s) so that the means and extremes below do not necessarily reflect the entire world experience.

This report therefore focalizes on a total of 62 patients (including a personal case), 61 females and 1 male. The mean age at presentation was 36 years (21-60 years), and most patients had lost a considerable amount of weight: the mean body mass index (BMI) was $25.5(19-30) \mathrm{kg} / \mathrm{m}^{2}$, the mean absolute weight loss was $64.1(22.4-121) \mathrm{kg}$, and the mean excess weight loss was $99.8 \%(69.3-109.3)$. The interval between RYGBP and intussusception varied between as short as 5 months and as long as 24 years, with a mean of 3.6 years. Roux-en- $Y$ gastric bypass had been performed using open surgery in 42 patients and laparoscopy in the other $20(32.2 \%)$.

The only common clinical finding at presentation was acute abdominal pain in all the patients. Nausea and/or vomiting was present in $73 \%$ of the patients, and three patients had hematemesis. Duration of symptoms before emergency room visit was between a few hours and 2 months, but in most cases less than 5 days. Some patients had had colicky intermittent abdominal pain before the current acute episode. Clinical examination was not conclusive in most patients, revealing tenderness but only rarely signs of peritoneal irritation. In six patients $(9.7 \%)$, however, a tender mass was noticed. The mean excess weight loss (EWL) in these six patients was 101.7, not different from the overall mean of $99.8 \%$. Only one patient had fever. Results of laboratory tests are rarely mentioned, and only ten $(16.1 \%)$ patients were found to have an elevated white blood cell count.

A variety of radiological tests were performed in these patients, including plain films, echography, upper GI series, and computerized tomography (CT) scan. No patient underwent MRI. Signs suggesting short bowel obstruction (dilated loops or air-fluid levels) were present in $93.5 \%$ of the patients. CT scan, which was performed in 33 patients, showed a "target sign" (Fig. 1), suggestive of intussusception [28] in 27 patients (81.8\%). Radiological tests were considered normal in only three patients, in whom the diagnosis was made during surgical exploration.

All patients underwent surgery. In six patients whose symptoms resolved spontaneously, surgery was delayed by a few days. The remaining patients were operated on emergently. A variety of intussusception types were noted at surgery: intussusceptions involving the jejunojejunostomy, including retrograde intussusceptions of the common limb into the biliopancreatic limb, or even all different limbs, and also intussusceptions distal to the jejunojejunostomy, or involving only the Roux limb, like in one of our cases. Although the exact anatomy of the intussusception is not always described, it is clear that the jejunojejunostomy 


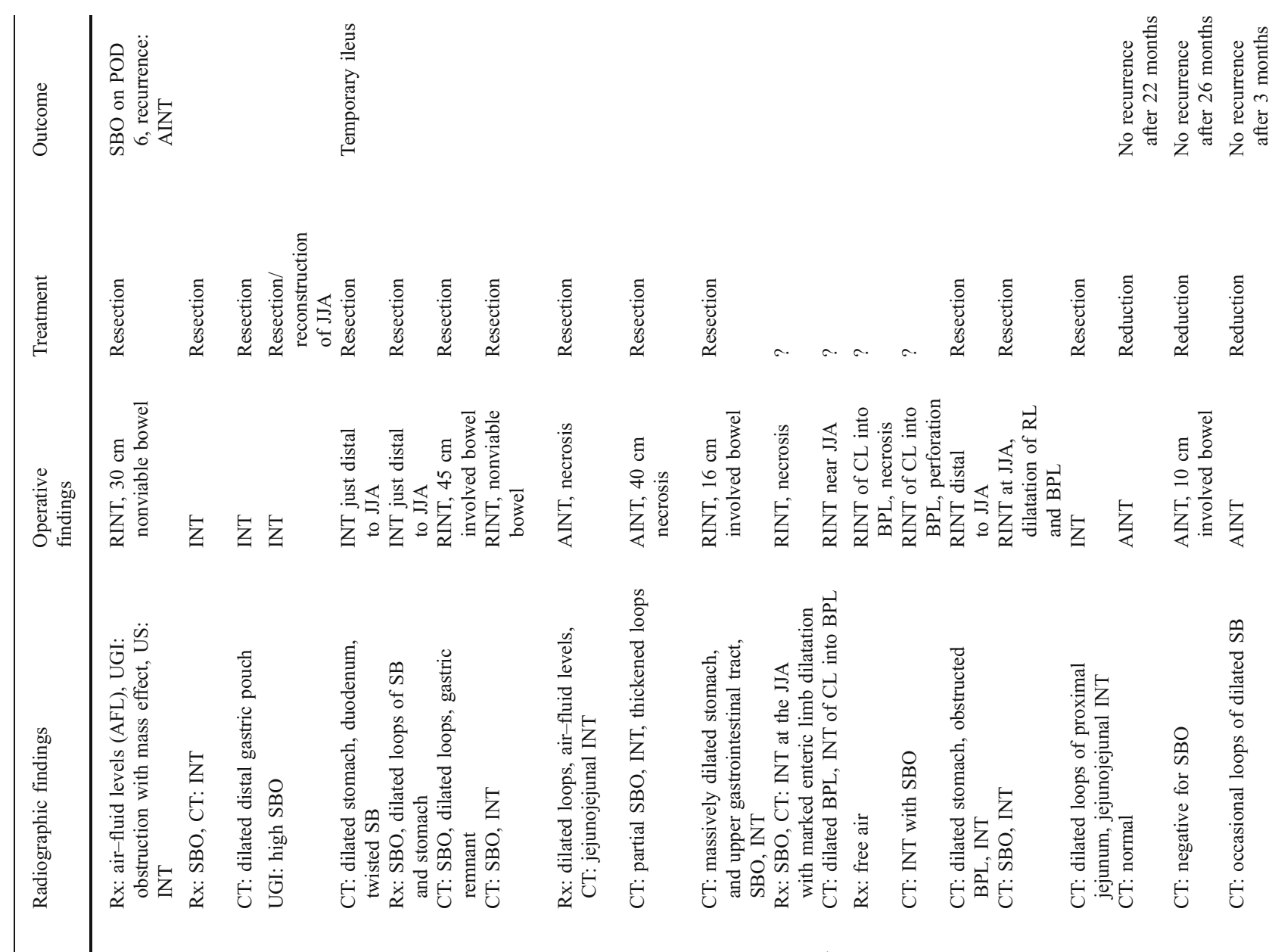

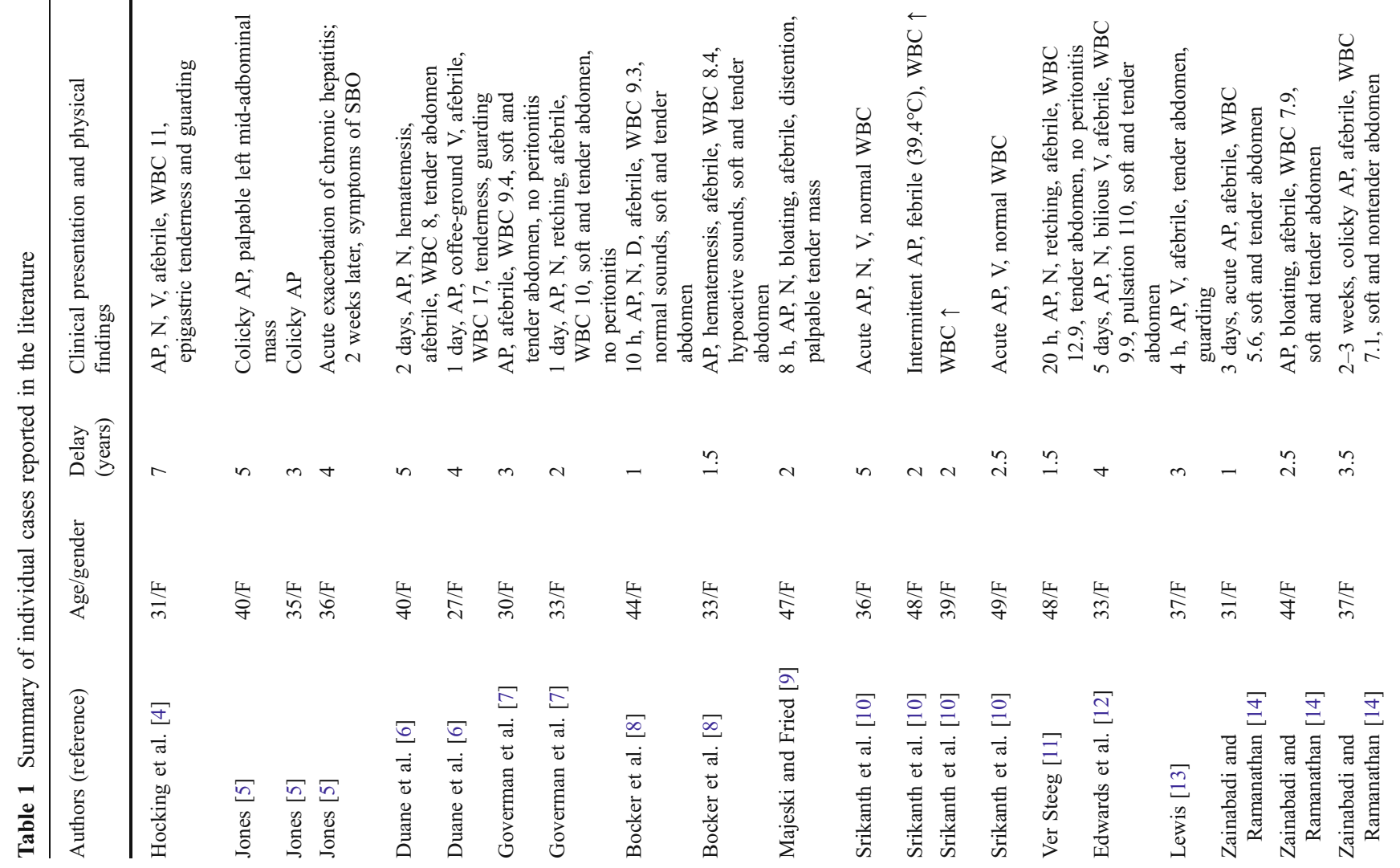




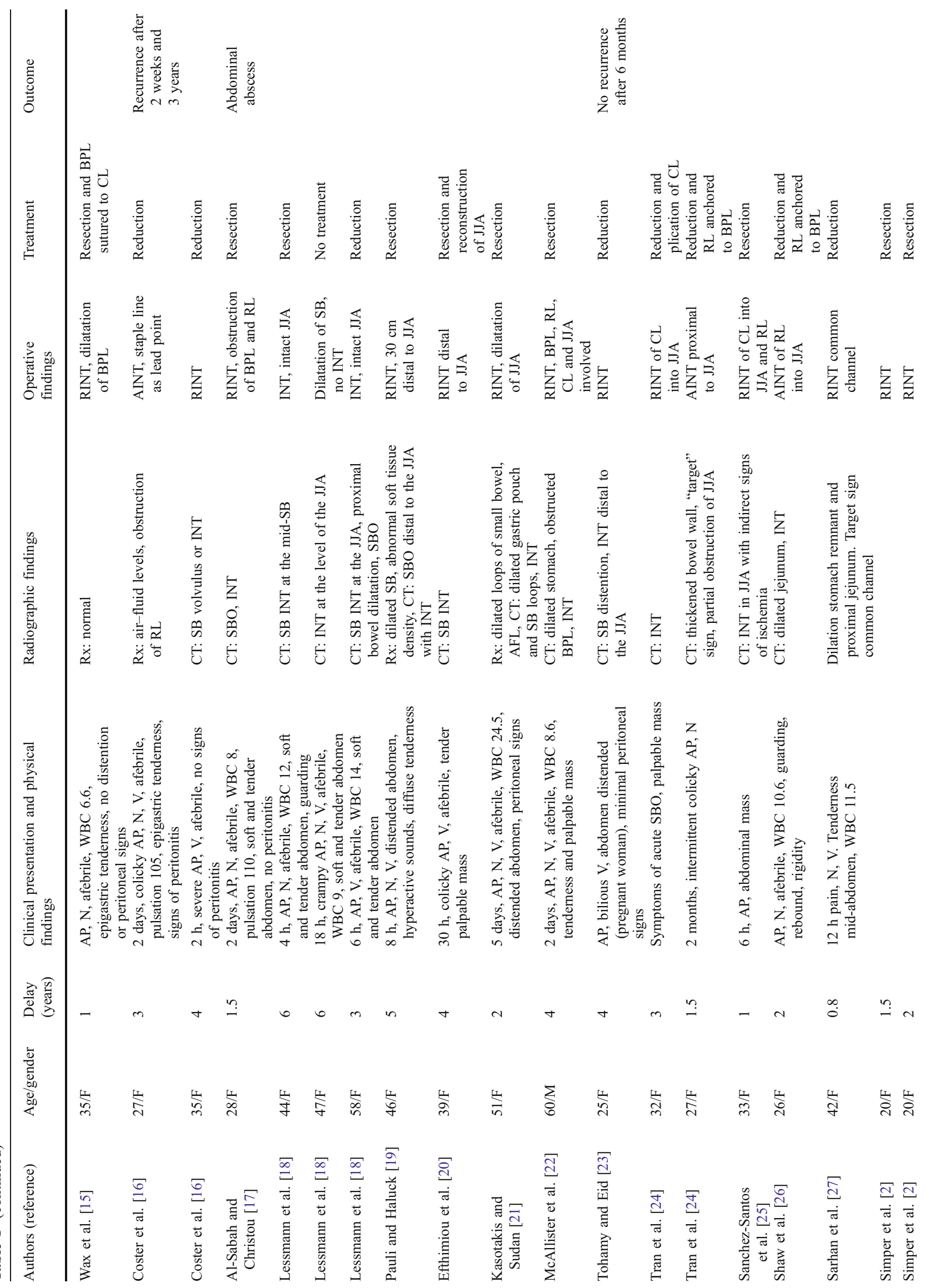




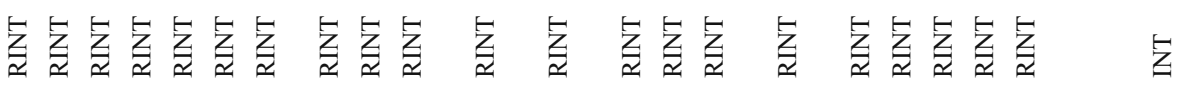

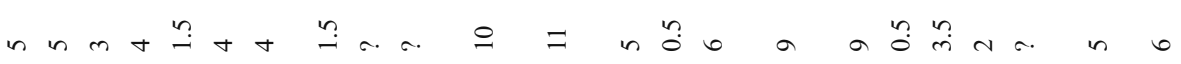

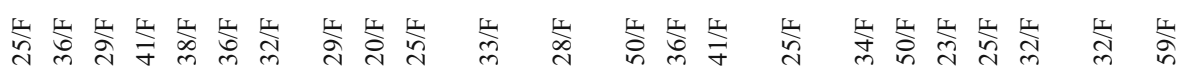

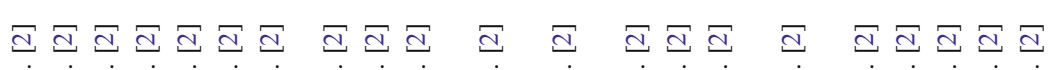

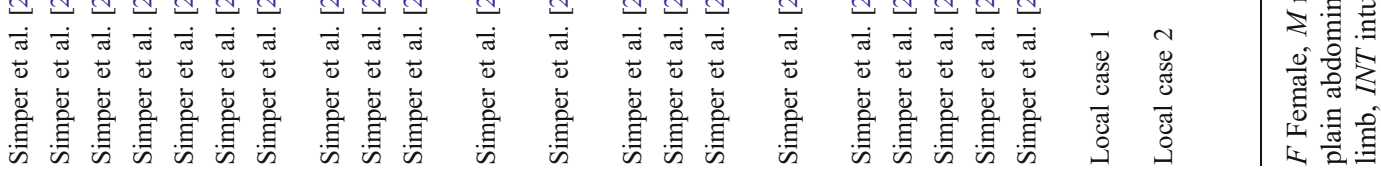


is involved in the vast majority of cases and that the commonest form is intussusception of the common channel into the jejunojejunostomy. A retrograde intussusception was found in $44(69.8 \%)$ patients and an antegrade intussusception in 8 (12.7\%). The type of intussusception was not mentioned in the other cases, and exploration showed no abnormality in the last patient, despite a typical preoperative $\mathrm{CT}$ scan with a target sign. In the latter patient, intussusception was considered to have resolved spontaneously [18]. An ischemic segment of jejunum was found in seven patients, including one with a perforation. Intussusception involved only a short segment of bowel, with a maximum of $45 \mathrm{~cm}$.

At surgery, small bowel resection of the invaginated segment was performed in $38(61.3 \%)$ patients, including those with ischemia. In three patients, this was coupled with reconstruction of the jejunojejunal anastomosis. Simple reduction of the invaginated segment was considered sufficient in $20(32.2 \%)$ patients although some kind of intestinal plication was added in 9 of them. Treatment is not specified for four patients and one local patient was treated conservatively. She still presents intermittent abdominal pain but has refused surgery so far.

Out of the 39 patients who underwent resection, 3 (7.7\%) presented with a recurrence between 1 and 7 months later. One of these patients, who initially had retrograde intussusception, developed the anterograde type the second time. At reoperation, reduction was performed, together with a Noble plication [4]. This patient later required a third operation because she developed an internal hernia between the sutures used for the plication, which was taken down. The second patient underwent plication the second time and still presents with intermittent abdominal pain. In the third one, the gastric bypass was completely taken down, anatomy was restored, and a sleeve gastrectomy was performed, with no subsequent complain [3]. Six (31.5\%) of the 19 patients with only reduction $+/-$ plication developed recurrence between 2 weeks and almost 3 years later. Five of these patients were reoperated and underwent resection the second time whereas the last one was treated conservatively. When compared with the recurrence rate after resection, the difference is statistically significant (31.5 versus $7.7 \%, p=0,04$, Fisher's exact test).

\section{Patient \# 1}

This 32-year-old woman presented 5 years after having undergone a laparoscopic RYGBP at our institution, with a 1-week history of pain in the left upper abdomen and nausea. Her BMI had dropped from 48.9 to $29.9 \mathrm{~kg} / \mathrm{m}^{2}$, and her body weight had been stable since more than 3 years. Clinical examination showed some abdominal tenderness, but no sign of peritoneal irritation. Blood tests, including a complete blood count, C-reactive protein, liver function tests, and pancreatic enzymes, were normal. CT scan (Fig. 1) showed a typical target sign suggestive of intussusception. Because she had no sign of obstruction and only mild findings at examination, she was observed, recovered fully, and was capable of tolerating a regular diet within 4 days. Since then, she presents similar episodes of intermittent abdominal pain, but has not returned to the emergency room and has refused an exploratory laparoscopy to rule out an internal hernia. Although highly likely, the diagnosis of intussusception was not proven in this patient, who did not undergo surgery, and she was therefore not included in the statistical analysis in this review. This case is reported here because, with intermittent episodes of colicky abdominal pain, she illustrates a possible, and probably rather common, form of presentation of transient intussusception or internal hernia. While CT may show dilated loops or even a dilated stomach remnant in both, it is unlikely to show a typical target sign in a case of an internal hernia and may prove useful for the differential diagnosis.

\section{Patient \# 2}

This 59-year-old lady had undergone an open Roux-en-Y gastric bypass in our hospital 6 years before presenting to the emergency room in 2006 with a 4-day history of crampy abdominal pain with vomiting. Her BMI was $20.7 \mathrm{~kg} / \mathrm{m}^{2}$ and physical examination showed abdominal tenderness and visible hyperactive dilated bowel loops. Because we suspected an internal hernia, we performed an exploratory laparoscopy, which did not reveal anything abnormal. On the first postoperative day, because she had persistent pain, she underwent a CT scan (Fig. 2), which showed a typical target sign, together with dilated and thickened jejunal loops, suggesting edema and ischemia. Laparotomy confirmed a jejunojejunal intussusception, just

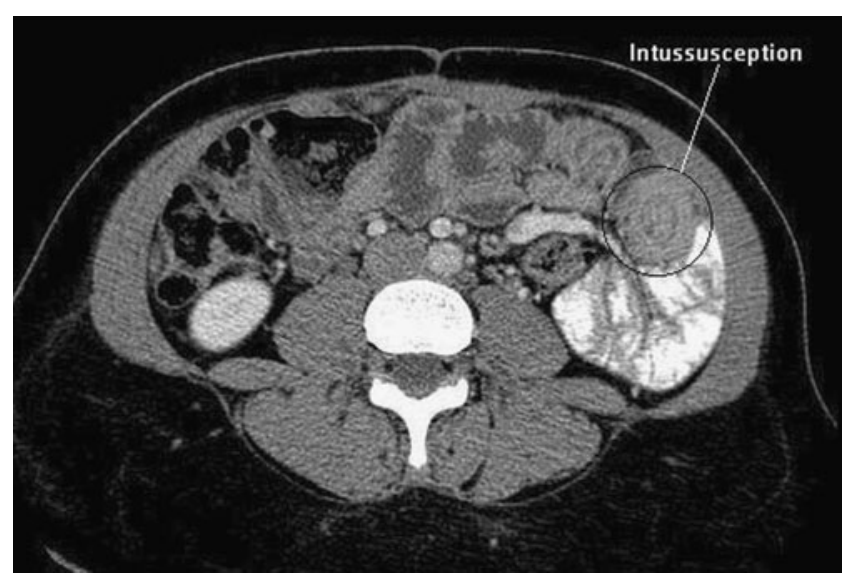

Fig. 1 CT scan showing a "target sign", suggestive of intussusception 


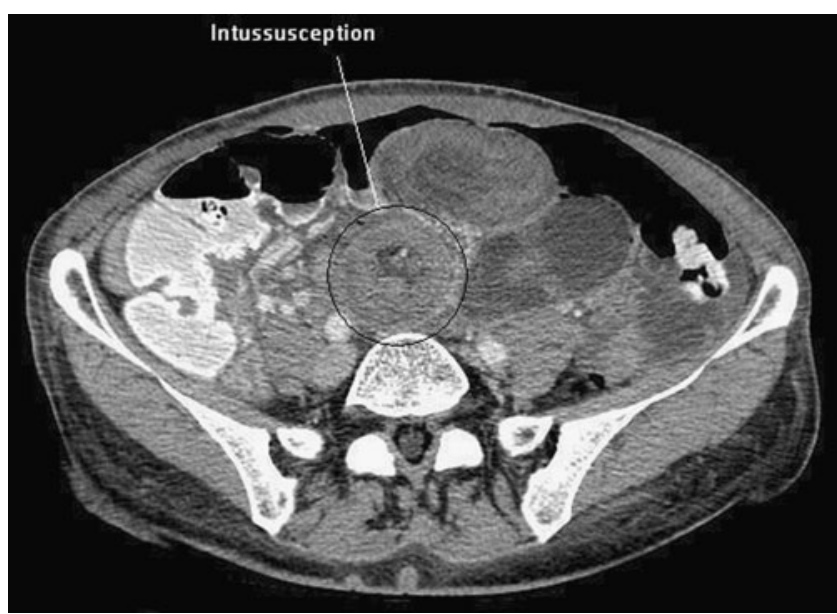

Fig. 2 CT scan showing a typical target sign, together with dilated and thickened jejunal loops, suggesting edema and ischemia

proximal to the jejunojejunostomy, which was reduced. As the bowel was viable, no resection was performed, but a few anchoring sutures were placed between the biliopancreatic limb and the Roux limb to prevent recurrence. The postoperative course was uneventful. Within the next 2 years, she came back to the emergency department several times with abdominal pain. Various tests were performed, with completely normal findings. In 2008, symptoms were more acute and lasted longer. A CT scan showed signs of partial obstruction and conservative treatment was attempted, but the symptoms recurred and a laparotomy was performed. A small palpable mass was noted in the distal Roux limb, and a small resection was performed, with uneventful recovery. Pathological analysis of the mass showed ectopic pancreatic tissue. Six months later, she came back to the emergency room with abdominal pain. CT scan showed an intussusception of the biliopancreatic limb near the jejunojejunostomy. At laparotomy, the bowel was considered viable after reduction, and a few anchoring sutures were placed again. During the following months, the patient had crampy abdominal pain every time she ate solid food and even sometimes with liquids. This was attributed to recurrent intermittent intussusception, and the patient was submitted to complete resection and reconstruction of her jejunojejunostomy, with end-to-side anastomoses of the biliopancreatic limb into the jejunum. Recovery was uneventful. She now eats normally and has not had further symptom.

\section{Discussion}

Prevalence

Small bowel intussusception is a rare long-term complication after Roux-en-Y gastric bypass. In the literature, the prevalence lies between $0.07 \%$ and $0.6 \%[2,6,7,15,20]$. In their large experience, Simper et al. [2] found 23 cases among 16,000 patients, for a prevalence of $0.15 \%$. More recently, the same group presented an enlarged series of 39 patients in the form of an abstract, but the new denominator was not available for calculation [3]. In our own experience, two cases among 1,200 patients represent $0.17 \%$. It is likely, however, that the true prevalence is slightly higher since intussusception can resolve spontaneously, so that some patients never present to the hospital, or the diagnosis is not made because symptoms disappear before any test is performed. The retrograde form of intussusception seems more frequent that the anterograde one and represents two thirds of reported cases, but intussusceptions can take several different forms and may involve all three limbs of the gastric bypass in various proportions.

Intussusception, both antegrade and retrograde, has been described on rare occasions after a variety of other digestive procedures involving a Roux-en-Y loop, including liver transplantation [29], pancreatic surgery [30, 31], biliary [32], esophageal surgery [33], and jejuno-ileal bypass [34]. To the best of our knowledge, no case has been reported after other bariatric procedures like gastric banding, vertical banded gastroplasty, sleeve gastrectomy, or even biliopancreatic diversions.

\section{Etiology}

In the adult, intussusception is usually anterograde and is associated with the presence of a "lead point", most often a small tumor or a polyp. As no such "lead point" was found in the cases that form this review, except in our second patient, the mechanism after Roux-en-Y gastric bypass must be different. Hocking et al. [4] were the first to suggest that intussusception in this setting might be related to small bowel motility disturbances. In the Roux limb of their patient, they found orad- and aborad-propagated migrating motor complexes, a minimal phase 2 motor activity, as well as the lack of conversion of motility to the "fed pattern" after a liquid meal. Normally, small bowel motility is initiated essentially in the duodenum, where the frequency of the pacesetter potential of the smooth muscle cells is fastest. These contractions are regular at rest and move distally at a speed of about $6-8 \mathrm{~cm}$ per minute. After a meal (fed state), intestinal motility is enhanced with contractions that mix the food with digestive juices and move the chime distally in an aborad direction. The capacity of the small bowel to convert to the fed state depends on the integrity of the vagal nerve, the presence of the duodenum, and the type of ingested food [35]. Transecting the jejunum to construct a Roux limb when doing RYGBP separates the distal jejunum from the duodenal pacemaker, which disrupts the propagation of the pacesetter 
potentials from the natural pacemaker into the Roux limb. This causes a drop in the pacesetter potential distal to the transection (i.e., in the Roux limb), allowing for ectopic pacemakers to arise in the Roux limb. These ectopic pacemakers generate pacesetter potentials, which migrate not only distally, but sometimes also in an orad direction, causing stasis and delaying emptying of the Roux limb. How these alterations cause intussusception is not entirely understood. It is postulated that the high pressure resulting from the meeting, at the jejunojejunostomy, of a normal peristaltic wave originating in the duodenum by the normal pacemaker with another one originating in the Roux limb from an ectopic pacemaker, acts as a lead point. While this might be true for intussusception arising at the level of the jejunojejunostomy, it does not explain why in some patients it develops along the Roux limb only, or only distal to the jejunojejunostomy. These motility disorders are also considered to be responsible for the various manifestations of the so-called Roux stasis syndrome, of which intussusception may represent the extreme stage [35].

The fact that no intussusception has been reported after biliopancreatic diversion (BPD) does not rule out this theory since division of the bowel in BPD occurs at a much more distal level, the Roux limb being formed by the ileum, which may lack pacemaker properties such as those mentioned above. In the only reported case of an intussusception after jejuno-ileal bypass, the jejunum was also divided proximally, and the blind limb invaginated was of the same configuration as the Roux limb in gastric bypass [35]. Others postulate that the suture line at the jejunojejunostomy acts as a lead point $[6,36]$. This was, however, not clearly demonstrated in most of the reported cases. Local inflammatory mediators resulting in nodal hyperplasia have also been mentioned as a possible cause [18], but this has not been substantiated yet.

Another fact that pleads in favor of motility disorders is that most patients affected by intussusception have lost a considerable amount of weight before they develop this complication. In this review, the mean EWL at presentation was $99.8 \%$, which is far superior to the mean EWL after standard RYGBP, which lies somewhere between $50 \%$ and $70 \%$ after 5 years. Motility disorders slowing emptying of the Roux limb could contribute to these patients eating less and hence to increased weight loss, by increasing their feeling of satiety. Furthermore, the thinner mesentery associated with increased weight loss might facilitate the development of intussusception, with less resistance related to the overall thickness of the intussuscepted segment. While motility disorders may play a role in extreme weight loss, intussusception, however, is unlikely to be responsible for it since the mean time from gastric bypass to intussusception is 3.6 years, well beyond the phase of weight loss that ends usually after about 18 months.

During the era of open surgery, adhesions were also considered a contributing factor to the development of intussusception. As this phenomenon also occurs after laparoscopic RYGBP ( $32.2 \%$ of the patients in this series), which is known to result in much fewer adhesions, the latter are unlikely to play a significant role.

The way the jejunojejunostomy is constructed does not seem to play a role either. Intussusceptions have been described after both the biliopancreatic limb aligned with the Roux limb in an isoperistaltic as well as anisoperistaltic way.

In this review, the vast majority of intussusceptions were reported in women $(62 / 63,98.4 \%)$. Although the denominator is unknown, this percentage by far outreaches the proportion of women undergoing RYGBP, which usually lies around $70-80 \%$, with $77 \%$ in our experience. This raises the possibility that sex and possibly unknown hormonal factors play a role in the etiology. This possibility has not been mentioned so far and warrants further research.

In summary, dysmotility disorders seem to be the most accepted basis for the development of intussusception after RYGBP, but the precise mechanism by which the latter develops remains to be elucidated. These motility disorders, with ectopic pacemakers, are typically found in the Roux stasis syndrome, a condition characterized by chronic abdominal pain, nausea, and vomiting; exacerbated by eating; and presented by some patients after a Roux-en-Y gastrojejunostomy.

\section{Risk Factors}

Interestingly, $98 \%$ of the reported patients are females. As mentioned before, this is much more than the usual percentage of operated patients in large bariatric series. On the basis of the available literature, however, there is no explanation why intussusception should predominate in women. Another interesting observation is that weight loss after RYGBP has been very important in most reported cases, with an average of $99.8 \% \mathrm{EWL}$, which translates into $64.1 \mathrm{~kg}$. In our series, as in many others [36, 37], the mean EWL after 4 years, the mean interval between RYGBP and intussusception, is around $65 \%$. Massive weight loss results in a very thin, but also elongated, mesentery. It could be a contributing factor to the development of intussusception in that a thin and long mesentery offers less resistance to invagination once the latter is initiated. One could also consider that intussusception and/or motility disorders in the alimentary limb could be the cause of extreme weight loss. We believe that this is unlikely to be the case as all but three patients in this review developed their intussusception well after the phase of rapid weight loss and weight stabilization, a mean of 3.6 years after gastric bypass.

\section{Clinical Presentation and Diagnosis}

The classic triad of intussusception in children, including abdominal pain, bloody stool, and a palpable mass, is rarely 
seen in adults. Symptoms are not specific. They can be acute, intermittent, or chronic. Abdominal pain is constant, and more than two thirds $(72 \%)$ of the patients present with nausea/vomiting. Duration of symptoms can vary from a few hours to a few months. Patients with complete intussusception and intestinal obstruction will have very acute symptoms $(<24 \mathrm{~h})$ whereas those with incomplete and very short intussusception may experience some pain, but no sign of obstruction. These patients may even have recurrent episodes before the diagnosis is made, like with internal hernia. Physical examination is most often not helpful. There is no fever. The abdomen is usually tender, but signs of peritonitis are unusual. A palpable mass, which is encountered only in one out of ten patients, should be suggestive. Blood tests should include a complete blood count, C-reactive protein, liver function tests, and pancreatic enzymes. Laboratory markers of inflammation or ischemia are often absent, which can be misleading [38]. In summary, the clinical picture is not alarming most of the time, and this is often also the case with other long-term complications of RYGBP, like internal hernia. In fact, if only the biliopancreatic limb is involved, the patient will present with abdominal pain only, sometimes with alarming signs like tachycardia and/or hypotension if necrosis is present, but there will be no clinical or radiological sign of obstruction, food and contrast flowing freely down the digestive tract. In these cases, pancreatic and/or liver function tests may be altered due to overdistension on the biliopancreatic limb, and the patient may falsely be diagnosed with acute pancreatitis. Internal hernia and intussusception, however, should always be included in the differential diagnosis of abdominal pain in patients with a history of RYGBP, and additional studies are always warranted. The possibility of intussusception should be mentioned especially in women since they represent $98.4 \%$ of the patients in this review. Since most patients have sustained important weight loss before intussusception occurs, the latter should be strongly suspected in patients with significant excess weight loss although it cannot be ruled out completely in those with less than ideal weight loss. One patient in this review had indeed lost only $22 \mathrm{~kg}$. Patients with severe acute abdominal pain after RYGBP should always be investigated promptly, bearing in mind that they can develop severe bowel obstruction with necrosis, both from internal hernia and intussusception. A plain abdominal radiograph can show signs of intestinal obstruction, but CT scan is the study of choice [28] and should be considered in all gastric bypass patients with severe acute or chronic abdominal pain of unknown origin $[12,39]$. CT scan can reveal dilated bowel loops and airfluid levels. It can also demonstrate the typical "target sign", which was present in $82 \%$ of the patients who had CT scan in this review. If only the biliopancreatic limb is involved, CT scan will show distension of the duodenum and stomach remnant. These signs are often overlooked by the nonspecialist or a radiologist who interprets the study without a bariatric surgeon's assistance. If they are left unrecognized, severe necrosis can develop and lead to the patient's death. When performed between episodes of pain in a patient with intermittent symptoms, however, CT scan can be totally normal. While it is very often helpful in the acute setting, CT scan is not entirely reliable if it proves negative in a gastric bypass patient with acute abdominal pain, especially with intussusception. A negative CT scan should not preclude surgical exploration, even many years after gastric bypass, since both internal hernia and intussusception can develop long after surgery and both can lead to bowel necrosis, sometimes with dramatic consequences.

\section{Treatment}

Although intussusception may resolve spontaneously, as in our first patients, treatment is usually surgical once the diagnosis is made, especially when clinical signs or paraclinical parameters suggest bowel necrosis. Conservative management is discouraged because it is hard to ascertain the absence of necrosis from the clinical and radiological signs alone, and because if intussusception persists, necrosis can develop at a later stage and lead to perforation [11]. If surgery is usually clearly indicated in the acute setting, the decision to operate on a patient with intermittent symptoms is more difficult. Recurrent acute and severe crampy or colicky abdominal pain after RYGBP, however, is due in most cases to a mechanical problem (internal hernia, bands/adhesions). Radiological imaging between episodes is unlikely to show any alteration, but will prove abnormal in most cases when performed during the symptoms. It may reveal signs of obstruction with various aspects suggestive of an internal hernia (abnormal course of mesenteric vessels, dilatation of bowel loops, or the whirl sign). Repeated episodes of pain, especially if very acute, should warrant surgical exploration. The latter must rule out an internal hernia, and all open mesenteric defects should be carefully closed with running nonabsorbable sutures. In the absence of internal hernia, however, and especially if a typical target sign was present on pre-exploration imaging, the most likely diagnosis is intussusception. Both internal hernia and intussusception can lead to isolated obstruction of the biliopancreatic limb, without the common clinical signs of intestinal obstruction (vomiting, absence of gas/ stool passage), and even without radiological evidence of obstruction (oral contrast progressing normally to the distal bowel). These signs need to be recognized, and this may require a bariatric surgeon or a radiologist who has experience with the complications of bariatric surgery. 
Laparoscopy or open surgery can be used, depending on the experience of the surgeon. There is no consensus as to which treatment is optimal for intussusception after RYGBP. If there is intestinal necrosis, the nonviable segment should be resected and a termino-terminal anastomosis performed. If the involved segment includes the jejunojejunostomy, the latter will need reconstruction. In the majority of patients, however, manual reduction of the invaginated segment is possible, and the bowel is viable. Simple reduction has been successfully reported by several authors $[14,16,18,23,26]$ whereas others, like us with our second patient, have observed recurrences, sometimes as early as 2 weeks after the first episode [2, 16]. Some surgeons have tried a variety of intestinal plications to limit the risk of recurrence, from a few anchoring sutures, as in our second patient, to a complete Noble enteropexy $[2,24]$. None of these techniques, however, has been able to achieve a complete recurrence-free outcome.

Several groups suggest that the invaginated segment should be resected whether or not it is viable $[2,4,10,12$, 20]. This seems logical if one assumes that intussusception is related to a lead point (ectopic pacemaker, staple line), as resection of the latter eliminates the cause. In this review, there is a statistically higher recurrence rate in patients who were treated by reduction $+/-$ plication/pexy when compared with patients treated by resection and anastomosis/ reconstruction of the jejunojejunostomy $(33.3 \%$ versus $7.7 \%, p=0.02$ ). Due to the limited number of reported cases and hence the experience of most individual surgeons with this rare complication, it is difficult to draw firm conclusions from these results. The aforementioned difference, however, strongly suggests that resection of the invaginated segment is the treatment of choice. Our own experience with our second patients supports this, and resection is also the treatment recommended by the group having the largest experience [2]. Recently, the same group has reported on five cases of gastric bypass reversal with conversion to a sleeve gastrectomy, with good clinical outcome regarding both weight maintenance and symptoms [3]. If resection is to be performed, however, and involves a portion of the alimentary limb, the surgeon should measure the length of the latter to make sure that it will still be long enough to prevent biliary reflux. If not, the Roux limb should be elongated, and the jejunojejunostomy reconstructed more distally.

When recurrence develops, treatment is even more controversial because of the very low number of reported cases. On the basis of this review, we conclude that resection with reconstruction of the jejunojejunostomy is the treatment of choice if intussusception recurs at or near the jejunojejunostomy. Reversal of the gastric bypass and conversion to another procedure like sleeve gastrectomy may be an option, but overall experience is insufficient to recommend it for the time being.

\section{Prevention}

As the exact pathophysiological mechanisms leading to intussusceptions after RYGBP are still unknown, it is difficult to suggest efficient preventing measures. Based on the theory that cutting the jejunum leads to motility disturbances in the Roux limb, Tu et al. [35] have designed an uncut Roux limb in which a musculo-serosal bridge of tissue is left between the gastrojejunostomy and the jejunojejunostomy on the mesenteric border while the mucosa is removed and the full-thickness antimesenteric portion is also excised. From a theoretical point of view, this could help the normal migrating motor complex, originating from the duodenal pacesetter, to progress normally along the jejunum at a normal frequency, thereby preventing the development of ectopic pacesetters in the Roux limb. This technique has been tested in dogs, and only a very slight decrease in the frequency has been demonstrated beyond the uncut portion. To the best of our knowledge, however, no application of this technique to humans has been reported so far.

\section{Conclusions}

Intussusception is a rare, but probably underreported, complication of Roux-en-Y gastric bypass. It may develop in an antegrade or retrograde direction, often near or at the level of the jejunojejunostomy. Clinical manifestations are usually acute, with abdominal pain with or without signs and symptoms of bowel occlusion. Some patients present with recurrent episodes of acute abdominal pain. Internal hernia should always be included in the differential diagnosis. The precise etiology of intussusception remains unknown, but the most accepted theory relies on the development of motility disorders following the division of the jejunum, which promote the emergence of ectopic pacesetters in the Roux limb, as seen in the Roux stasis syndrome. Symptoms and clinical signs are unspecific. CT scan represents the diagnostic test of choice [28] and shows the typical target aspect in about $80 \%$ of the patients. Treatment in the acute setting should probably involve resection of the affected segment, with anastomosis. If the jejunojejunostomy is affected, the latter should be resected and reconstructed. Simple reduction and fixation seems to lead to an inadmissibly high recurrence rate, and we would discourage it on the basis of this review although no method can guarantee the absence of any recurrence. Patients with recurrent acute abdominal pain after RYGBP should be explored surgically to exclude a potential internal hernia. If none is found, reconstruction of the jejunojejunostomy may be considered if CT scan performed during an acute episode shows a typical target sign, since intussus- 
ception, according to this review, involves the jejunojejunostomy in the vast majority of cases and is likely to recur.

Conflict of Interest The authors declare that they have no conflict of interest in relation to the present manuscript.

\section{References}

1. Buchwald H, Oien DM. Bariatric/metabolic surgery worldwide 2008. Obes Surg. 2009;19:1605-11.

2. Simper SC, Erzinger JM, McKinlay RD, et al. Retrograde (reverse) jejunal intussusception might not be such a rare problem: a single group's experience of 23 cases. Surg Obes Relat Dis. 2008;4:77-83.

3. Simper S, Erzinger J, McKinlay R, et al. Laparoscopic reversal of gastric bypass with sleeve gastrectomy for the treatment of recurrent Rint (retrograde intussusception). Obes Surg. 2009;19:977.

4. Hocking MP, McCoy DM, Vogel SB, et al. Antiperistaltic and isoperistaltic intussusception associated with abnormal motility after Roux-en-Y gastric bypass: a case report. Surgery. 1991;110:109-12.

5. Jones KB. Biliopancreatic limb obstruction in gastric bypass at or proximal to the jejunojejunostomy: a potentially deadly, catastrophic event. Obes Surg. 1996;6:485-93.

6. Duane TM, Wohlgemuth S, Ruffin K. Intussusception after Roux-enY gastric bypass. Am Surg. 2000;66:82-4.

7. Goverman J, Greenwald M, Gellman L, et al. Antiperistaltic (retrograde) intussusception after Roux-en-Y gastric bypass. Am Surg. 2004;70:67-70.

8. Bocker J, Vasile J, Zager J, et al. Intussusception: an uncommon cause of postoperative small bowel obstruction after gastric bypass. Obes Surg. 2004;14:116-9.

9. Majeski J, Fried D. Retrograde intussusception after Roux-en-Y gastric bypass surgery. J Am Coll Surg. 2004;199:988-9.

10. Srikanth MS, Keskey T, Fox SR, et al. Computed tomography patterns in small bowel obstruction after open distal gastric bypass. Obes Surg. 2004;14:811-22.

11. Ver Steeg K. Retrograde intussusception following Roux-en-Y gastric bypass. Obes Surg. 2006;16:1101-3.

12. Edwards MA, Grinbaum R, Ellsmere J, et al. Intussusception after Roux-en-Y gastric bypass for morbid obesity: case report and literature review of rare complication. Surg Obes Relat Dis. 2006;2:483-9.

13. Lewis AM. A 37-year-old with vomiting and severe, left-sided abdominal pain 3 years after gastric bypass surgery. J Emerg Nurs. 2006;32:48-50.

14. Zainabadi K, Ramanathan R. Intussusception after laparoscopic Roux-en-Y gastric bypass. Obes Surg. 2007;17:1619-23.

15. Wax JR, Wolff R, Cobean R, et al. Intussusception complicating pregnancy following laparoscopic Roux-en-Y gastric bypass. Obes Surg. 2007;17:977-9.

16. Coster DD, Sundberg SM, Kermode DS, et al. Small bowel obstruction due to antegrade and retrograde intussusception after gastric bypass: three case reports in two patients, literature review, and recommendations for diagnosis and treatment. Surg Obes Relat Dis. 2008;4:69-72.

17. Al-Sabah S, Christou N. Intussusception after laparoscopic Roux-en-Y gastric bypass. Surg Obes Relat Dis. 2008;4:205-9.
18. Lessmann J, Soto E, Merola S. Intussusception after Roux-en-Y gastric bypass for morbid obesity. Surg Obes Relat Dis. 2008;4:664-7.

19. Pauli EM, Haluck RS. Antiperistaltic (retrograde) intussusception after laparoscopic Roux-en-Y gastric bypass procedure. Surg Obes Relat Dis. 2008;4:567-8.

20. Efthimiou E, Court O, Christou N. Small bowel obstruction due to retrograde intussusception after laparoscopic Roux-en-Y gastric bypass. Obes Surg. 2009;19:378-80.

21. Kasotakis G, Sudan R. Retrograde intussusception after Roux-enY gastric bypass for morbid obesity. Obes Surg. 2009;19:381-4.

22. McAllister MS, Donoway T, Lucktong TA. Synchronous intussusceptions following Roux-en-Y gastric bypass: case report and review of the literature. Obes Surg. 2009;12:1719-23.

23. Tohamy AE, Eid GM. Laparoscopic reduction of small bowel intussusception in a 33-week pregnant gastric bypass patient: surgical technique and review of literature. Surg Obes Relat Dis. 2009;5:111-5.

24. Tran D, Halmi D, Kolesnikov E. Intussusception following Roux-enY gastric bypass: two case reports and literature review. Obes Surg. 2009;8:1038.

25. Sanchez-Santos R, Gonzalez S, Lopez N, et al. Acute abdomen following laparoscopic Roux-en-Y gastric bypass: retrograde intussusception in jejunojejunostomy. Obes Surg. 2009;8:1034.

26. Shaw D, Huddleston S, Beilman G. Anterograde intussusception following laparoscopic Roux-en-Y Gastric bypass: a case report and review of the literature. Obes Surg. 2010;20(8):1191-4.

27. Sarhan M, Leaque A, Miegge L. Retrograde intussusception after Roux-en-Y gastric bypass. Am Surg. 2010;76:454-6.

28. Gayer G, Zissin R, Apter S, et al. Adult intussusception-a CT diagnosis. Br J Radiol. 2002;75:185-90.

29. Shin N, Hasegawa K, Ikeda M, et al. Adult intussusception induced by the stump of the jejunal loop after liver transplantation. Hepatogastroenterology. 2008;55:898.

30. Whipple OC, Stringer EF, Senkowski CK, et al. Retrograde intussusception of the efferent limb after a pancreatico-jejunostomy. Am Surg. 2003;69:353.

31. Gigena M, Villar HV, Knowles NG, et al. Antegrade bowel intussusception after remote Whipple and Puestow procedures for treatment of pancreas divisum. World J Gastroenterol. 2007; 13:5954.

32. Chen CC, Huang SC, Huang SC, et al. Retrograde intussusception as a complication of Roux-en-Y anastomosis for choledochal cystreport of one case. Zhonghua Minguo Xiaoer Keyi Xuehui Zazhi. 1994;35:546.

33. Ozdogan M, Hamaloglu E, Ozdemir A, et al. Antegrade jejunojejunal intussusception after Roux-en-Y oesophagojejunostomy as an unusual cause of postoperative intestinal obstruction. Surg Today. 2001;31:355.

34. Baylem NJ, Pounaras D, Ramus I (2010) An extremely late complication of Roux-en-Y jejunoileal bypass. Obes Surg. doi:10.1007/s11695-009-0023-4

35. Tu BN, Kelly KA. Motility disorders after Roux-en-Y gastrojejunostomy. Obes Surg. 1994;4:219-26.

36. Wittgrove AC, Clark GW. Laparoscopic gastric bypass, Roux-enY-500 patients: technique and results, with 3-60 month follow-up. Obes Surg. 2000;10:233-9.

37. Linner JH, Drew RL. Why the operation we prefer is the Roux-Y gastric bypass. Obes Surg. 1991;1:305-6.

38. Agha FP. Intussusception in adults. AJR Am J Roentgenol. 1986;146:527-31.

39. Azar T, Berger DL. Adult intussusception. Ann Surg. 1997;226:134-8. 